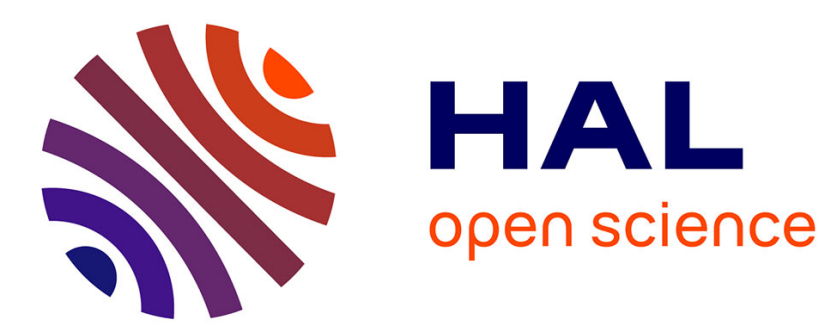

\title{
Structuration rituelle de la relation défunts-vivants au Cambodge dans les morts individuelles et collectives
}

\author{
Anne Yvonne Guillou
}

\section{To cite this version:}

Anne Yvonne Guillou. Structuration rituelle de la relation défunts-vivants au Cambodge dans les morts individuelles et collectives. L'Autre, 2018, Morts ou vifs, 19 (2), pp.267 - 275. 10.3917/lautr.057.0267 . halshs-02531604

\section{HAL Id: halshs-02531604 \\ https://shs.hal.science/halshs-02531604}

Submitted on 3 Apr 2020

HAL is a multi-disciplinary open access archive for the deposit and dissemination of scientific research documents, whether they are published or not. The documents may come from teaching and research institutions in France or abroad, or from public or private research centers.
L'archive ouverte pluridisciplinaire HAL, est destinée au dépôt et à la diffusion de documents scientifiques de niveau recherche, publiés ou non, émanant des établissements d'enseignement et de recherche français ou étrangers, des laboratoires publics ou privés. 
Structuration rituelle de la relation défunts-vivants au Cambodge dans les morts individuelles et collectives

L'Autre, cliniques, cultures et sociétés, 2018, volume 19, numéro 2, pp. 267-275

Anne Yvonne Guillou

DOI https://doi.org/10.3917/lautr.057.0267

https://www.cairn.info/revue-l-autre-2018-3-page-267.htm 
Anne Yvonne Guillou est anthropologue, chargée de recherche au CNRS, en poste pour I'IRASEC au Cambodge. Actuellement, ses recherches portent principalement sur les constructions (notamment rituelles) des mémoires collectives et des processus de résilience sociale après les destructions et les violences de masse au Cambodge et en Asie du Sud-Est. Ces thèmes couvrent le champs de l'anthropologie religieuse et notamment celui l'étude du bouddhisme khmer et du système religieux khmer dans son ensemble.

Cette recherche a été financée par le Centre national de la recherche scientifique (CNRS) via le Centre Asie du Sud-Est (CNRS-EHESS-INALCO) et I'Institut de recherche sur l'Asie du Sud-Est contemporaine (CNRS-MAEDI).

Institut de recherche sur l'Asie du Sud-Est contemporaine (IRASEC)

4th Floor, 179 Thanon Witthayu, Lumphini, Pathum Wan, Bangkok 10330, Thailand

anne.guillou@cnrs.fr 
RESUME

L'auteur examine les différentes phases de la mort en milieu khmer, au Cambodge, où le bouddhisme theravada officiellement pratiqué est nourri d'un ancien fonds animiste. La mort y est perçue comme une transition plutôt qu'une rupture finale. L'importance fondamentale de la terre et, à un moindre degré, de l'eau et du feu, est soulignée concernant les rituels funéraires. L'article décrit la "carrière du mort " c'est-à-dire les phases rituellement construites qui jalonnent la transformation du cadavre en défunt puis en ancêtre. Cette "carrière " est parallèle au processus de deuil des vivants et structure celui-ci. Plus encore, la présence des défunts dans la communauté et leur commerce avec les humains obéissent à des règles précises, de façon à ce qu'une relation structurée, non invasive, s'établisse. La dernière partie de l'article évoque le cas des victimes du régime khmer rouge (1975-1979) qui disposent d'un statut que les Khmers n'assimilent pas à celui des morts de malemort traditionnels.

MOTS CLE : BOUDDHISME ;KARMA ; ANIMISME ; RITUEL ; INCINERATION ; DEUIL ; CADAVRE ; KHMERS ROUGES ; TERRE ; MALEMORT

\section{SUMMARY}

The author examines the different phases of death among the Khmer in Cambodia where Theravada Buddhism, which is the official religion, is influenced by the old animist background. Death is perceived as a transition rather than a final breakdown. The crucial importance of the earth and to a lesser extent of water and fire, in funerary rituals is underlined. The article describes the "career of the dead", i.e. the ritual phases which have to be passed through during the transformation of the corpse into a defunct person and then an ancestor. This "career" goes along with the mourning process of the living and is structured by it. Moreover, the presence of the dead among the living and their relationship with them is governed by clear rules so as to be well structured, and non invasive. The last part of the article is about the victims of the Khmer Rouge regime (1975-1979). Unexpectedly, the Khmer do not assimilate them into the traditionnal category of the "dead who died a bad death".

KEY WORDS: BUDDHISM; KARMA; ANIMISM; RITUAL; CREMATION; MOURNING; CORPSE; KHMER ROUGE; EARTH; BAD DEATH

La autora examina las diferentes fases de la muerte en el entorno jemer en Camboya, en donde el budismo Theravada, religión oficial, se alimenta de un antiguo fondo animista. La muerte es percibida como una transición más que como una ruptura final. La importancia fundamental de la tierra y, en menor grado, del agua y el fuego, es resaltada en los rituales funerarios. El artículo describe la "carrera del muerto", es decir, las fases construidas ritualmente que jalonan la transformación del cadáver en difunto y luego en ancestro. Esta "carrera" es paralela al proceso de duelo de los vivos y lo estructura. Además, la presencia de difuntos en la comunidad y su relación con los humanos obedecen a reglas precisas, de manera que una relación estructurada y no invasiva se establece. La última parte del artículo evoca el caso de las víctimas del régimen jemer rojo (1975-1979), las cuales disponen de un estatus que los jemeres no asimilan al de los muertos de una mala muerte tradicionales.

PALABRAS CLAVE: BUDISMO, KARMA, ANIMISMO, RITUAL, INCINERACIÓN, DUELO, CADÁVER, JEMERES ROJOS, TIERRA, MALA MUERTE 
Il y a quelques années, au cours de l'une des discussions, nombreuses et riches, que nous eûmes, Geneviève Welsh ${ }^{1}$ et moi-même, à propos de ses patients cambodgiens réfugiés en France, elle me parla de l'un d'entre eux, monsieur Prah². Après des années de mise en confiance, celui-ci s'était mis à lui décrire ce qu'il estimait être la source de ses troubles, à savoir ses cauchemards envahis par les morts qu'il avait vus pendant la guerre ; et d'autre part, les visites que lui rendaient une bonne dizaine d'êtres invisibles. Si les défunts effrayants de ses rêves restaient anonymes, M. Prah était capable de nommer (par leur nom personnel) les autres êtres invisibles.

L'examen de la liste des noms personnels des esprits, fournis par M. Prah, révéla qu'ils étaient tous connus au Cambodge. Ils font partie de l'environnement familier de la plupart des $\mathrm{Khmers}^{3}$ et fréquentent de façon tout à fait habituelle certaines catégories de médiums. II s'agissait donc de comprendre - du point de vue de l'anthropologue, tout au moins - pourquoi le commerce avec ces êtres invisibles devenait la source des frayeurs et des troubles de M. Prah. Une partie de la réponse, avons-nous conclu Geneviève Welsh et moi-même, se trouvait dans la forme de la relation que M. Prah entretenait avec (ou se voyait imposer par) eux : ils surgissaient de façon impromptue, envahissante, anarchique et souvent inamicale. Les conditions d'un rapport normal aux esprits - du point de vue de la société khmère - n'étaient pas réunies. Restaient à déterminer en quoi consistait celui-ci.

$C^{\prime}$ est à cette question que s'attache à répondre cet article, en me limitant, toutefois, aux défunts, ceux-ci ne constituant qu'une catégorie de l'ensemble des êtres invisibles ${ }^{4}$, qui peuplent l'univers khmer. En effet, il n'y a pas de différence ontologique entre les multiples esprits, les défunts et les humains vivants. Seule leur apparence varie. Ainsi, par exemple, certains esprits tutélaires de territoire sont issus de défunts, qui sont eux-mêmes d'anciens humains vivants. Le terme khmoc est d'ailleurs le terme générique désignant en langue khmère les " esprits " de toutes catégories aussi bien que le cadavre lui-même ; ce qui est en accord tant avec la vision bouddhiste de la vie qu'avec l'ontologie animiste, lesquelles participent toutes deux du système de représentation cambodgien.

La recherche que j'expose ici met en évidence les caractéristiques des relations entre morts et vivants dans les principaux contextes du décès, c'est-à-dire celui de la mort individuelle quand elle est considérée comme normale (bonne mort) ou tragique (malemort) ; et celui de la mort en masse (mort collective) dont les Cambodgiens ont fait l'expérience à plusieurs moments de leur histoire, et particulièrement au cours du génocide perpétré par le régime khmer rouge (1975-1979) dont l'administration de la mort possède les mêmes caractéristiques que celle des autres régimes totalitaires et génocidaires. Les relations entre la communauté des vivants et les morts évoluent, au Cambodge comme ailleurs, au fil du temps - un temps qui est scandé par le devenir du défunt luimême, depuis sa lente dégradation physique jusqu'à son accession, dans le meilleur des cas, au statut d' « ancêtre ${ }^{5}$.

Les analyses présentées dans cet article s'appuient sur le retraitement d'un matériau ethnographique relatif aux morts et à la mort, recueilli au cours de plusieurs recherches de terrain en anthropologie médicale, en anthropologie religieuse et en anthropologie de la mémoire, s'étendant de 1990 à 2017 (soit plus de huit ans au total). J'ai particulièrement utilisé les données des monographies de deux villages (province de Pursat, Ouest du Cambodge) recueillies de 2007 à 2017 (vingt mois d'enquête), complétées par des données recueillies dans d'autres provinces (région de Phnom Penh, Battambang, Oddar Meanchey, Kompong Thom). Je les ai complétées par l'observation de funérailles en mai 2017 dans la province de Prey Veng (Sud-Est du Cambodge) que j'ai comparées aux quatres autres cérémonies funéraires complètes auxquelles j'ai assisté-participé dans le passé au Cambodge (ou parmi les Cambodgiens réfugiés en France, dans un cas). Je ne reviens pas ici sur les

1 Psychiatre, psychanalyste (SPP) et consultante à l'Association de santé mentale du $13^{\text {ème }}$ arrondissement de Paris.

${ }^{2}$ Il s'agit d'un pseudonyme. Voir la contribution de Geneviève Welsh sur M. Prah dans ce volume.

${ }^{3}$ Les Khmers constituent environ $85 \%$ de la population du Cambodge.

${ }^{4}$ Pour une recension des êtres invisibles qui peuplent le Cambodge, voir Ang (1986).

${ }^{5}$ " Ancêtre » désigne ici, non pas le fondateur d'une lignée mais un défunt familial ou villageois protecteur. 
spécificités de la méthode de l'enquête ethnographique, basée sur l'immersion totale, la connaissance approfondie de la langue et le recoupement des informations fournies par l'observation (directe, photographique, filmique), les discussions et les entretiens formels. Je ne reviens pas non plus sur la très abondante littérature anthropologique traitant des relations entre enquêteur et enquêtés.

\section{La mort comme processus et comme transition}

Au Cambodge comme dans la plupart des sociétés, la mort constitue moins un événement rapide, individuel (ou familial) et marquant une fin irrémédiable, qu'un processus lent - transition plutôt que destruction - se déroulant sur des mois ou des années et affectant l'ensemble de la communauté.

Le schéma général du processus de séparation du mort et des vivants, établi par Hertz (19056), s'y applique également. La dégradation du corps physique constitue une période intermédiaire et potentiellement dangereuse car des éléments encore présents de la dépouille peuvent se manifester avec plus ou moins de violence auprès des vivants. La dissolution de l'ancien corps conditionne la formation du corps nouveau (ou de l'existence nouvelle), dans un autre monde (ou dans celui-ci, dans le cas du Cambodge) car la réincarnation d'un élément vital est la règle plutôt que l'exception. Lors des funérailles finales ${ }^{6}$, le défunt accède au nouvel état $d^{\prime}$ " ancêtre ». La force maléfique qui émanait de lui lors de la période de transformation physique durant laquelle il ne pouvait se détacher entièrement du monde des humains, se transforme en force bénéfique, "pacifiée " (Hertz 1905-6) grâce aux rituels funéraires pratiqués. II se meut alors, en fonction du rang qui fut le sien, en force protectrice de sa famille ou de sa communauté. "La cérémonie finale redonne une vie sociale au mort " (Hertz 19056). A ce moment, vivants et morts se séparent.

La " carrière du mort ${ }^{7}$, c'est-à-dire les phases rituellement construites qui jalonnent la transformation du cadavre en défunt puis en ancêtre, est parallèle au processus de deuil des vivants et structure celui-ci. Plus que cela, le groupe social est pétri de ses rapports aux défunts, quelle que soit la forme que ces derniers acquerront. Par ailleurs, la présence des défunts dans la communauté et leur commerce avec les humains obéissent à des règles précises, de façon à ce qu'une relation structurée, non invasive, s'établisse. Ce sont ces règles que je $m$ 'attacherai à décrire plutôt que les rituels funéraires, riches et complexes (Leclère 1907 ; Bizot 1981; Ang et al. 2014 ; Davis 2016) en euxmêmes.

\section{La désagrégation de l'enveloppe physique et l'évolution du statut du mort}

Commençons pas la carrière d'un mort « normal » au Cambodge. Un mort de bonne mort, ici comme dans d'autres sociétés, est celui dont le décès n'est ni violent ni prématuré (Baptandier 2001). C'est celui dont le " fil de la vie » n'a pas été " rompu », qui a épuisé son content de vie. L'exemple parfait est celui d'un vieillard qui finit son existence en pieux bouddhiste et part doucement de la " maladie des personne âgées ". S'il existe de nombreux termes pour désigner la mort, l'on dit fréquemment - notamment dans le nord-ouest du Cambodge - d'une telle personne morte de sa belle mort qu'elle est khūc (« pourrie »). Semblable à un fruit, elle a connu toutes les étapes de la maturation

${ }^{6}$ Certaines sociétés pratiquent, d'une façon ou d'une autre, les doubles funérailles (enterrement, déterrement et incinération).

7 Le terme de " carrière du mort " s'inspire de l'ancienne et abondante littérature anthropologique consacrée aux défunts, dont la recension dépasse largement les limites de cet article. Plus particulièrement, il s'appuie à la fois sur le concept de " carrière » défini par Erving Goffman (1968) et que je retravaille dans le sens de la « biographie sociale » du défunt, et de la mise en parallèle de la transformation du cadavre avec celle de la communauté des vivants, que l'on doit à Robert Hertz (1905-1906). 
jusqu'à la dernière puis elle reprendra forme dans ce monde ou un autre car l'ensemble des rituels funéraires incluent une symbolique de la renaissance (Bizot 1981 ; Ang et al. 2014).

L'état mental qui précède la mort est fondamental pour le devenir du défunt. II abordera son voyage dans les meilleures conditions s'il accepte de se détacher sereinement de ce monde sans peur, sans haine, sans attachement excessif à l'égard de ce et ceux qu'il y laisse. D'ailleurs, bien avant la mort, de nombreux cinquantenaires ou soixantenaires - en particulier des femmes - se préparent progressivement à leur départ en fréquentant les monastères bouddhiques les jours saints et en multipliant les actes méritoires.

L'agonisant est accompagné par les prières des moines, des officiants rituels laïcs et de l'entourage qui mettent en avant le principe bouddhique majeur de l'impermanence, aidant ainsi le mourant à quitter ce monde. Ce principe, rappelé à tous à de multiples reprises au cours des funérailles pour soulager le chagrin des proches, veut que tout être, toute pensée, tout sentiment, toute production conditionnée soient appelés à disparaître, à se transformer. Ainsi, par exemple, lors de funérailles auxquelles j'ai assistées en mai 2017, les deux sentences suivantes étaient inscrites sur chaque côté du cercueil : " naissance - vieillesse/maladie - mort " et, faisant plus directement référence aux fondements de la doctrine bouddhique "l'état corporel [est associé à] l'impermanence ; l'état corporel [est associé à] la soif de vivre/souffrance ; l'état corporel [est associé au] non-soi ». Chacun s'efforce de contenir ses manifestations de tristesse pour faciliter le voyage que doit entreprendre la personne décédée.

La période intermédiaire, avant la transformation du cadavre par le feu et/ou la terre, est délicate car certains éléments vitaux du défunt restent présents sous diverses formes. Il existe une certaine assimilation actuelle entre la notion pré-bouddhique $d$ ' " esprits vitaux ", au nombre théorique de dix-neuf, qui animent le corps et le mental, et la notion bouddhique de viññanna (conscience, perception, sensation), laquelle est censée disparaître après la mort. Après son dernier souffle, le mort, dans ses différentes composantes physiques et non-physiques, passe par différents états qui sont scrutés par les vivants comme autant de signes de ce que sera la relation entre l'un et les autres. Cependant, le risque, en compagnie d'un mort de bonne mort, ne dépasse pas celui de la présence trop envahissante d'un défunt qui se détache avec difficulté et pourra d'ailleurs renaître à proximité sous une forme humaine (Prigent 2015) ou animale (cette dernière n'est pas souhaitée mais demeure de toute façon inconnaissable aux vivants). Si l'on traçait un continuum des relations entre mort et vivants, nous aurions, à l'une des extémités, l'archétype du mort partant facilement et rapidement. En position intermédiaire se situeraient les morts qui restent proches des vivants dans leurs affects et leurs besoins physiques. Ils communiquent par l'intermédiaire de rêves où ils formulent leurs demandes, lesquelles sont très proches de celles des vivants (nourriture, boisson, cigarettes, vêtements). D'ailleurs, des offrandes de nourriture continuent à être faites jusqu'à la cérémonie du septième jour suivant le décès. Dans le cas des funérailles de mai 2017 , le défunt s'est manifesté à l'aube suivant la nuit de la crémation par la présence d'une grosse sauterelle verte grimpant au rideau du dai sous lequel avait lieu la prière. Enfin, à l'extrémité de l'échelle, d'autres manifestations, plus brutales, sont attribuées aux morts de malemort. J'ai ainsi assisté, dans les circonstances dramatiques du décès d'un jeune homme de dix-huit ans dans un accident de voiture, à la possession "spontanée " $^{8}$ d'un voisin par le jeune homme décédé. Le voisin est venu, la nuit suivant le drame, hurler et pleurer à la porte de la mère du défunt, décrivant en détail les minutes précédant l'accident tout en faisant rugir le moteur d'une moto. Cette mise en scène a aidé la mère à comprendre ce qu'il s'était passé et à répéter encore et encore pendant des jours, les détails de l'accident dans une épreuve cathartique qui semble l'avoir aidée à surmonter la perte.

\section{INSERER PHOTO 1}

Le corps est habituellement incinéré en milieu urbain (sauf dans les familles se réclamant d'ancêtres chinois proches) mais la crémation est souvent précédée d'un enterrement de plusieurs

${ }^{8}$ Possession spontanée signifie ici que la monture du défunt n'était pas habituellement un médium. 
années dans les villages, notamment ceux du Nord-Ouest. La justification de l'enterrement est la nécessité de retarder la crémation jusqu'au moment où la somme d'argent nécessaire à la cérémonie sera réunie. Ces "doubles funérailles " sont souvent collectives, par souci d'économie, entend-on fréquemment. Mais ces rationalisations économiques cachent la nécessité profonde du contact du corps avec la terre, avec le sol, dans le traitement funéraire paysan ; une nécessité de plus en plus remises en question par un bouddhisme soumis, depuis une dizaine d'années, à un mouvement profond d'orthodoxification et privilégiant l'incinération directe.

La pratique de l'enterrement - et ce qu'implique le contact avec la terre - s'insère dans un système de représentations à différentes strates. Dans chacune d'entre elles, la notion de " terre » décline une connotation différente du terme : terre comme substance et " matière élémentaire ", conception religieuse du sol et, enfin, territoire local ou national. La première raison pour laquelle l'enterrement est préféré à l'incinération rapide, souvent donnée par les Khmers auxquels j'ai posé la question en diverses circonstances, est que les morts craignent la brûlure du feu tandis qu'ils apprécient la fraîcheur de la terre (la fraîcheur est une vertu cardinale). Dans la région d'Angkor, Ang et al. (2014 : 73) soulignent la nécessité que «Brah Dharaṇī [la déesse de la terre]embrasse [le mort] ». La terre est également l'un des éléments qui constituent le corps humain (avec l'eau, le feu et l'air) et le seul à ne pas disparaître. On entend parfois dire aussi que les morts nourrissent la terre, la font pousser, lui est nécessaire - et inversement. Plus profondément encore, " eau [et] terre » est le terme sentimental habituel par lequel les Khmers désignent leur pays et, de fait, ces deux éléments interviennent dans les cérémonies funéraires. D’autres données ethnographiques en plus de celles-ci suggèrent que la communauté khmère à l'échelle nationale est aussi faite du corps de ses enfants.

Une étape supplémentaire est franchie avec la cérémonie du septième jour après laquelle, pourrait-t-on dire, le mort se dégage de son enveloppe physique et devient un défunt. En cas d'incinération, les restes conservés sont déposés dans une urne. Les offrandes de nourriture cessent et le riz non décortiqué, disposé dans un plat posé près du corps puis près du bûcher ou de la tombe, est dispersé. Le centième jour, marqué par une autre cérémonie, transforme le mort en " ancêtre ". II pourra éventuellement exercer une protection particulière de ses descendants. Sous le régime khmer rouge, certaines personnes disent par exemple avoir demandé et obtenu la protection d'un parent ou d'un grand-parent (le plus souvent maternel mais paternel également) décédé. A ces deux occasions, les moines sont invités à réciter des prières et un grand banquet réunit les parents, les amis et les villageois.

\section{INSERER PHOTO 2}

\section{Carrière du défunt : un voyage inconnaissable mais accompagné par les vivants}

II n'y a guère que les anthropologues et certains moines érudits pour se poser la question de ce qu'il se passe exactement après le centième jour. La réponse est maintenue dans un flou nécessaire car les diverses sources d'influence culturelle des Khmers n'apportent pas la même réponse au problème. En effet, le système religieux khmer est construit sur la base d'influences diverses hindouisme, bouddhisme tantrique, mahayana et theravada qui sont venus irriguer à partir des premiers siècles de l'ère chrétienne (et à partir du 14ème s. pour le theravada) un ensemble plus ancien que l'on pourrait qualifier approximativement d'animiste au sens de Descola (2005) dans lequel les êtres humains cohabitent avec d'autres êtres (animaux, plantes, pierres, sources, objets mais aussi esprits divers dont certains ont été des êtres humains...) dont ils partagent l'intériorité (sentiments, idées, volonté..) en dépit d'apparences extérieures dissemblables. Selon la conception bouddhiste khmère, le défunt se désagrège entièrement. II n’y a pas de reste. La renaissance est causée par la soif 
de vivre du défunt - une sorte de force qui le retient à la vie et le conduit à la renaissance. La forme de cette renaissance dépendra de ses actes (pensées, actions, volition...) passés. Mais tout cela est inconnaissable par les humains vivants et nul ne sait si ses proches feront un séjours aux enfers bouddhiques, resteront errer sur terre ou bien entreront rapidement dans le cycle des renaissances. La forme nibbanique du bouddhisme (accent mis sur l'extinction) n'est pas présente au Cambodge qui privilégie les formes kammatique (accent mis sur le karma) et apotropaïques (accent mis sur les vertus magiques du bouddhisme) (Spiro 1982). En d'autres termes, la renaissance est souhaitée. Cela est également plus compatible avec les représentations animistes selon lesquelles les " fantômes " sous différentes formes restent parfois dans l'environnement des vivants.

Dans la mesure où le karma des défunts pèse de tout son poids dans leur capacité de renaissance et la qualité de celle-ci, l'opération majeure au cours de toutes les cérémonies calendaires qui suivent est l'envoi de mérites bouddhiques aux défunts. L'opération consiste pour les vivants à réaliser l'acte méritoire par exellence : l'offrance à un moine. Le bilan positif de cette bonne action que l'on appelle le mérite bouddhique, est alors adressé au défunt dans le but d'élever son bilan karmique. Ainsi, dans le bouddhisme khmer, les défunts sont plus présents que la doctrine bouddhique ne l'énonce.

Autre entorse au bouddhisme doctrinal, la cérémonie annuelle la plus importante au Cambodge, avec le Nouvel An (avril), est la Quinzaine des Morts durant le mois luni-solaire de septembre-octobre. Durant ce rituel riche en symboles et en significations multiples, les défunts sans exception viennent à la rencontre des vivants, le jour (plutôt réservé aux relations avec les défunts familiers) comme la nuit (temps privilégié des morts inconnus, des âmes errantes). Une légende dit que les défunts se rendent, lors de ce grand rendez-vous, dans sept monastères en espérant y rencontrer leurs parents. A l'issue de la Quinzaine, ils sont invités à repartir dans leur monde à l'aide de petites barques chargées d'offrandes (dans certaines provinces).

\section{Les victimes du régime khmer rouge}

Or depuis la fin des années 1970, ces lois sociales générales sont confrontées à un événement historique majeur, celui des destructions massives et des morts de malemort en grand nombre. Les destructions, commencées par les bombardements étatsuniens sur le sol cambodgien lors de la guerre du Vietnam, ont atteint leur acmée sous le Kampuchéa Démocratique (" régime khmer rouge », 19751979), un régime maoïste révolutionnaire qui s'est efforcé de construire par la terreur une nouvelle société. Il a fait environ 1,7 million de victimes (soit près d'un quart de la population cambodgienne de 1975) mortes de faim, de maladies non soignées, d'épuisement ou exécutées. Une guerre civile, opposant des factions hostiles au gouvernement installé militairement par le Vietnam, a succédé à ce génocide. La paix n'est véritablement revenue au Cambodge qu'en 1998, après la mort de Pol Pot.

II s'agit donc de comprendre quel type de traitement a été appliqué aux victimes, sans entrer dans les détails des différentes productions mémorielles qu'a suscitée les expériences collectives et individuelles de ces destructions - en particulier celle très particulière du régime khmer rouge (Guillou

2012). Je n'évoquerai ici que les réponses villageoises, non institutionnelles et non gouvernementales.

Les victimes de la révolution khmère rouge entrent a priori dans la catégorie des morts de malemort. Or les personnes décédées avant l'heure, sans avoir pris conscience - grâce aux rituels et aux prières notamment - de leur fin, font des morts dangereux comme si tout le potentiel de vie qu'ils détenaient encore se déversait de façon incontrôlée dans la nature et sur les vivants. Aux morts de malemort ne sont offerts que des funérailles hâtives. Leurs restes sont éloignés des habitations et les spécialistes religieux renforcent les protections rituelles de l'entourage. 
Toutefois, le génocide khmer rouge a produit une mort différente : collective, de masse, anonyme, dans un contexte de malheur généralisé ${ }^{9}$. II a suscité une réponse spécifique qui ne s'inscrit pas totalement dans la prise en charge des morts de malemort individuels.

Le comportement habituellement reconnu aux morts de malemort, à savoir leur harcèlement malveillant des vivants, cède la place, dans le cas des victimes du régime khmer rouge à une autre représentation : celle de morts en souffrance, qu'il convient d'aider, dont il convient de prendre soin plutôt que de s'en protéger. Au cours de mes enquêtes à proximité des lieux de mort collective (prisons politiques, infirmeries, lieux de massacres), les villageois m'ont décrit des manifestations de fantômes, par des lumières, des bruits dans la nuit. Mais au fil des années, ces lieux où erraient de tels esprits sont devenus "fades " $(s \bar{a} p)$ selon l'expression khmère, c'est-à-dire inactifs. J'ai également fréquemment entendu dire que les personnes décédées durant cette période étaient trop affaiblies pour se transformer en fantômes capables de nuire aux vivants. Le potentiel d'action des morts est en effet toujours proportionnel au « capital » qui était le leur lorsqu’ils étaient en vie.

Quand les Cambodgiens ont retrouvé une partie de leur liberté de culte et de mouvement, vers la fin des années 1980, les morts délaissés ont bénéficié de deux sortes de traitements.

D’abord le traitement habituel des défunts familiaux, qui consiste à leur adresser des mérites bouddhiques par l'entremise des moines afin d'élever leur devenir karmique (rituel dit pan்sukūl). Contrairement aux Vietnamiens, lancés depuis une vingtaine d'années dans une recherche fiévreuse des corps de leurs défunts disparus pendant la guerre américaine (Kwon 2008), les Khmers peuvent organiser ce rituel sans le support physique de la dépouille humaine. Cela leur a été d'un grand secours pour venir en aide aux centaines de milliers de disparus. Le rituel pańsukūl s'adresse à la sorte d'énergie qui sera propulsée après la mort, selon son bagage positif ou négatif, vers des mondes supérieurs et des formes supérieures d'existence ou vers les mondes infernaux et des formes d'existence inférieure tels celles des animaux. Ces pan்sukūl sont réalisés avec le plus grand faste durant les cérémonies annuelles (Quinzaine des Morts, Nouvel An) mais les parents peuvent à tout moment organiser ce rituel en présence d'un moine. J'ai ainsi rencontré des familles qui venaient dans les monastères les plus proches des lieux présumés du décès de leurs proches pour organiser ce rituel de partage de mérites. Ces offrandes aux moines accompagnées d'une prière spécifique soulage considérablement les vivants et leur permet d'exprimer leur chagrin en entrant en communication (indirecte) avec leurs disparus. Car le bien-être des survivants dépend de celui des défunts. C'est là le point fondamental de l'économie affective du deuil chez les Khmers.

D’autre part, les ossements anonymes ont été rassemblés soit à la demande des autorités dans les années qui ont suivi la fin du régime khmer rouge (début des années 1980) soit à la propre initiative des villageois vivant à proximité de charniers. Parfois des habitants ont rêvé qu'un mort de ces charniers réclamait des soins sous forme de nourriture. Ils ont parfois organisé eux-mêmes des crémations informelles puis ont invité ces "voisins " inconnus à rejoindre les monastères ou leur village d'origine. Conformément aux représentations de la carrière des défunts au Cambodge, ces défunts, bien qu'anonymes, sont propiciés au moyen d'offrandes afin qu'ils se transforment en esprit protecteur.

Dans certains cas, les défunts convertis en esprit protecteur prennent la forme d'un véritable esprit tutélaire de territoire ( $a n a k t \bar{a})$, sorte d'ancêtre (au sens de premier habitant des lieux), dit " maître de l'eau et de la terre ", qui veille sur le lieu où ils ont fini leur vie et sur ses habitants vivants. Ceux-ci ont soin en retour de les honorer lors des grandes cérémonies calendaires, voire, plus régulièrement, lors des quatre jours saints mensuels du calendrier bouddhique.

Le système rituel et religieux khmer a donc été à même de prendre en charge les victimes du génocide khmer rouge, qui ont été réintégrés rituellement dans le monde commun des défunts selon leur catégorie karmique (défunts en chemin vers la renaissance, âmes errantes) et sociale (morts

${ }^{9}$ Sur les spécificités de la mort collective en contexte français, voir Clavandier (2004). 
familiaux et morts anonymes). Sitôt la liberté de culte retrouvée au Cambodge dans les années 1980 ainsi que dans tous les pays de l'exil, les Khmers ont partagé une profusion de mérites avec leurs défunts par l'intermédiaire des moines. Leur effort maximal s'est porté sur le rehaussement de la carrière de leurs morts afin de leur permettre d'accéder à la forme de vie la plus élevée possible. Les vivants sont en perpétuelle dette envers leurs parents et l'organisation de ces cérémonies permet d'exprimer gratitude et affection. Elles constituent l'expression culturellement acceptée et structurée de la douleur de la perte.

\section{Références}

Ang C. Les êtres surnaturels dans la religion populaire khmère,. Paris : CEDORECK ; 1986.

Ang C, Preap C, Sun C. Damnnoe jīvit manussa khmaer moeul tām pidhī chlan் văy. Phnom Penh : Yosothor ; 2014. [Les cycles de vie des Cambodgiens vus du point de vue des rites de passage]

Bizot F. Le don de soi-même. Recherches sur le bouddhisme khmer, vol. III, Paris, Publications de l'EFEO ; 1981.

Baptandier B. directeur. De la malemort en quelques pays d'Asie. Paris : Karthala ; 2001.

Clavandier G. La mort collective. Pour une sociologie des catastrophes. Paris : CNRS Editions ;

2004.

Davis E. Deathpower. Buddhism's ritual imagination in Cambodia. Columbia Univ. Press : New York ; 2016.

Descola P. Par-delà nature et culture. Paris : Gallimard ; 2005.

Goffman E. Asiles. Paris : Editions de Minuit ; 1968 [1 ère éd. am. 1961].

Guillou A Y. Temporalité et définitions des corps après le génocide khmer rouge. In : AnstettGessat E, Dreyfus JM, directeurs. Cadavres impensables, cadavres impensés. Approches méthodologiques du traitement des corps dans les violences de masse et les génocides. Paris : Petra ; 2012, p. 107-118.

Guillou A Y. An alternative memory of the Khmer Rouge genocide : the dead of the mass graves and the land guardian spirits (neak ta). South East Asia Research. 2012; 20(2): 193-212. Disponibilité sur

Internet $<$ http://www.ingentaconnect.com/content/ip/sear/2012/00000020/00000002/art00004\#expand/col lapse>

Hertz R. La représentation collective de la mort. Année sociologique, 1905-1906 ; 1re série, T. $X: p .48-137$.

Kwon H. Ghosts of War in Vietnam. Cambridge University Press: Cambridge; 2008.

Leclère A. La crémation et les rites funéraires au Cambodge. Crémation de sa Majesté Noroudam Roi du Cambodge. Hanoi : F.H. Schneider Imprimeur-éditeur ; 1907.

Prigent S. Ces enfants qui se souviennent de leur vie antérieure. Convocations de certains défunts et nouages de partenariats à Thnaot Chum (Cambodge). L'Homme. 2015; 213(1): 69-92. Disponibilité sur Internet: <https://www.cairn.info/revue-I-homme-2015-1-page-69.htm>

Spiro ME. Buddhism and Society. A great tradition and its Burmese vicissitudes. Berkeley: University of California Press; 1982. [1ère ed. Am. 1970].

Welsh G. Les fantômes au travail. L'autre, Cliniques, Cultures et Sociétés 2018; 19 (3). 


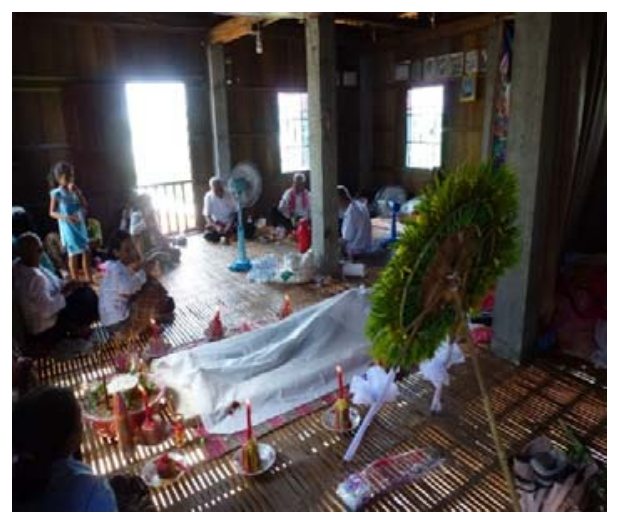

PHOTO 1 - Offrande dite du "paddy avec banlai [sorte de gingembre] » aux pieds du défunt. Cette offrande est faite à la sage-femme au cours de la cérémonie de dation du nom au nouveau-né. Symbolise la renaissance. Second jour du décès. Province de Prey Veng, Cambodge, mai 2017. Photo de l'auteur.

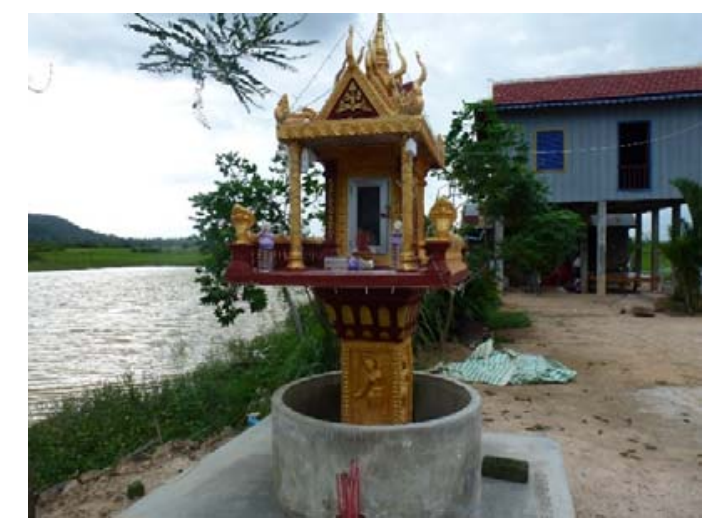

PHOTO 2. Au 7ème jour, les restes de la crémation sont conservés dans une urne. Ici, l'urne a été placée dans un autel au dieu du sol, dont le pilier baigne dans un bassin contenant des poissons. Le lien au sol et à l'eau est symboliquement entretenu même en cas de crémation. Province de Prey Veng, Cambodge, mai 2017. Photo de l'auteur. 